\title{
Quantitative Evaluation of the Fetal Cerebellar Vermis Using the Median View on Two-Dimensional Ultrasound
}

\author{
Cenk Gezer, ${ }^{1, *}$ Atalay Ekin, ${ }^{1}$ Naciye Sinem Gezer, ${ }^{2}$ Ulas Solmaz, ${ }^{1}$ and Mehmet Ozeren ${ }^{1}$ \\ ${ }^{1}$ Department of Obstetrics and Gynecology, Izmir Tepecik Training and Research Hospital, Izmir, Turkey \\ ${ }^{2}$ Department of Radiology, Faculty of Medicine, Dokuz Eylul University, Izmir, Turkey \\ "Corresponding author: Cenk Gezer, Department of Obstetrics and Gynecology, Izmir Tepecik Training and Research Hospital, Izmir, Turkey. Tel: +90-5325239130, E-mail: \\ drcenkgezer@gmail.com
}

Received 2015 November 19; Revised 2016 January 15; Accepted 2016 January 25.

\begin{abstract}
Background: Evaluation of the cerebellum and vermis is one of the integral parts of the fetal cranial anomaly screening. Objectives: The aim of this study was to create a nomogram for fetal vermis measurements between 17 and 30 gestational weeks. Patients and Methods: This prospective study was conducted on 171 volunteer pregnant women between March 2013 and December 2014. Measurements of the fetal cerebellar vermis diameters in the sagittal plane were performed by two-dimensional transabdominal ultrasonography.

Results: Optimal median planes were obtained in 117 of the cases. Vermian diameters as a function of gestational age were expressed by regression equations and the correlation coefficients were found to be highly statistically significant $(\mathrm{P}<0.001)$. The normal mean ( \pm standard deviation) for each gestational week was also defined.

Conclusion: This study presents the normal range of the two-dimensional fetal vermian measurements between 17 and 30 gestational weeks. In the absence of a three-dimensional ultrasonography, two-dimensional ultrasonography could also be used confidently with more time and effort.
\end{abstract}

Keywords: Cerebellum, Fetus, Prenatal Diagnosis, Ultrasound, Vermis

\section{Background}

Evaluation of the cerebellum and vermis is one of the integral parts of the fetal cranial anomaly screening $(1,2)$. The entire fetal vermis can be visualized by ultrasound from 18 weeks of gestation (3). Main evaluation of the fetal cerebellum, vermis and cisterna magna is performed with the serial axial planes and slight angulations between them (4). Additional coronal and sagittal planes are usually required in high-risk pregnancies to rule out several fetal cerebellar anomalies (5).

Vermian agenesis is an uncommon condition (3). It may be isolated or associated with other malformations such as Dandy-Walker malformation and its variants (1). However, vermian agenesis can be complete or partial and the severity of vermian hypoplasia has been demonstrated to correlate significantly with the severity of mental retardation $(6,7)$.

In the evaluation of fetal vermis with ultrasound, the traditional axial planes may cause false appearance of vermian dysgenesis (8). Therefore, mid-sagittal imaging which displays the entire vermis sufficiently is needed. Although its demonstration may take time and effort, using median plane enables to visualize the craniocaudal and an- teroposterior diameters of the cerebellar vermis, vermian lobules, fourth ventricle, pons, and the corpus callosum (9).

As misinterpretation of the axial scanning plans causes diversity between the prenatal diagnosis and autopsy findings, reference values of sagittal vermian diameters are crucial for clinical practice (8).

\section{Objectives}

The aim of this study was to create a nomogram for fetal vermis measurements between 17 and 30 weeks of gestation.

\section{Patients and Methods}

\subsection{Study Population}

This prospective study included 171 volunteer pregnant women with singleton fetuses who admitted to our perinatology unit from March 2013 to December 2014. The study was performed in accordance with the ethical standards laid down in the declaration of Helsinki and the research 
protocol was approved by the Independent bioethics committee for scientific research of our center. Informed consent was obtained from all participants. Pregnancies with estimated fetal weight and measurements within the normal range for gestational age, history of regular menses with accurate date of the last menstrual period and available measurements of the crown rump length in early pregnancy accordant with fetal age were included in the study. The pregnancies with chronic maternal diseases, fetal chromosomal and structural anomalies and growthretarded fetuses were excluded from the study.

\subsection{Data Collection}

Fetal vermian diameters were obtained during the routine ultrasound examinations which were performed either to rule out fetal anomalies or for the fetal follow-up between 17 and 30 weeks of gestation with a Logic 5 Pro (general electric health care, Milwaukee, USA) ultrasound machine by using a $5 \mathrm{MHz}$ curvilinear transducer. Each patient was examined by one examiner (C.G) in order to eliminate interobserver variability. Measurements were performed on a median slice for demonstration of the vermis, brainstem, fourth ventricle, and cisterna magna (Figure 1). Each measurement was performed at least twice and the average of the two values was noted. Craniocaudal (CC) diameter was measured from the culmen superiorly to the uvula inferiorly, and the anteroposterior (AP) diameter from the central lobule anteriorly to the tuber posteriorly (10).

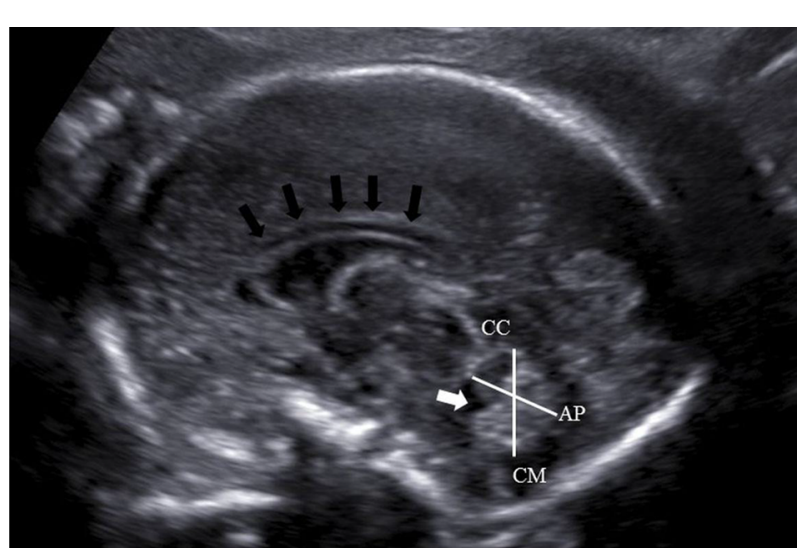

Figure 1. Measurement of the cerebellar vermian anteroposterior (AP) and craniocaudal (CC) diameters. CM, cisterna magna; white arrow, fourth ventricle; black arrows, corpus callosum.

\subsection{Statistical Analysis}

The means and standard deviations of the vermian parameters were calculated and regression analysis of the vermian $\mathrm{AP}$ and $\mathrm{CC}$ diameters as a function of gestational age was performed. Collected data were assessed by using Shapiro Wilk's test for normality. A P value less than 0.05 was considered to indicate statistical significance. All statistical tests were performed using the SPSS software, version 21.0 (IBM Corp. released 2012. IBM SPSS statistics for windows, version 21.0. Armonk, NY: IBM Corp.).

\section{Results}

A total of 171 fetuses between 17 and 30 weeks of gestation were included in the study. Fourteen cases were excluded from the study since the estimated fetal weight and measurements were out of the normal range for gestational age. During the ultrasonographic examinations, an optimal median plane could be obtained in 117 of 157 cases. In 40 of 157 fetuses (25.5\%), the measurements could not be achieved because the acoustic shadowing from the skull hindered visualization of the posterior fossa.

The mean maternal age of the study group was 27.1 (range, 17 - 44) years and the female to male ratio of the fetuses was 1.14. The regression equations for vermian CC, AP diameters, surface area, and transcerebellar diameter (TCD) modelled as a function of gestational age were: CC diameter $(\mathrm{cm})=(0.091 \times$ gestational age $)-0.777$, AP diameter $(\mathrm{cm})=(0.075 \times$ gestational age $)-0.668$, TCD diameter $(\mathrm{cm})=(0.165 \times$ gestational age $)-1.271$, surface area $\left(\mathrm{cm}^{2}\right)=$ ( $0.164 \times$ gestational age $)-2.489$. Pearson correlation coefficients of gestational age with vermis CC diameter, AP diameter, surface area, and TCD were 0.961, 0.965, 0.957, and 0.974 , respectively (All P values $<0.001$ ) (Figures 2 - 5); AP diameter $=0.965(\mathrm{P}<0.001)($ Figure 3$)$; surface area $=0.957$ $(\mathrm{P}<0.001)$ (Figure 4); and TCD $=0.974(\mathrm{P}<0.001)$ (Figure 5).

Table 1 presents the calculated mean \pm SD values, and Table 2 presents the 5th, 50th, and 95th centiles of transcerebellar and vermian diameters by gestational age between 17 and 30 weeks of gestation. Ratios of TCD to vermian diameters at each gestational week are shown in Table 3 .

\section{Discussion}

The embryogenesis of the cerebellar vermis begins rostrally in the midline during the ninth gestational week. The formation of the vermis antedates that of the cerebellar hemispheres by 30-60 days, and the entire vermis and cerebellum are complete by the end of the 15th week (11). The ultrasonographic studies report the complete formation of the vermis by the end of the 16 th week in agreement with the embryologic studies (12). 
Table 1. Values of Cerebellum and Cerebellar Vermis at Each Gestational Week ${ }^{\mathrm{a}}$

\begin{tabular}{|c|c|c|c|c|c|c|}
\hline Gestational Age, $w$ & Number of Fetuses & Mean Gestational Age, w & CC Diameter, cm & AP Diameter, cm & Surface Area, cm $^{2}$ & $\mathrm{TCD}, \mathrm{cm}$ \\
\hline 17 & 6 & 17.5 & $0.79 \pm 0.06$ & $0.63 \pm 0.11$ & $0.43 \pm 0.06$ & $1.69 \pm 0.08$ \\
\hline 18 & 9 & 18.3 & $0.84 \pm 0.08$ & $0.67 \pm 0.10$ & $0.48 \pm 0.10$ & $1.78 \pm 0.08$ \\
\hline 19 & 7 & 19.5 & $0.99 \pm 0.08$ & $0.78 \pm 0.05$ & $0.77 \pm 0.15$ & $1.98 \pm 0.13$ \\
\hline 20 & 10 & 20.5 & $1.04 \pm 0.07$ & $0.85 \pm 0.04$ & $0.85 \pm 0.12$ & $2.11 \pm 0.09$ \\
\hline 21 & 11 & 21.4 & $1.16 \pm 0.08$ & $0.95 \pm 0.04$ & $0.94 \pm 0.11$ & $2.23 \pm 0.06$ \\
\hline 22 & 9 & 22.3 & $1.30 \pm 0.14$ & $1.07 \pm 0.11$ & $1.19 \pm 0.23$ & $2.38 \pm 0.12$ \\
\hline 23 & 8 & 23.6 & $1.41 \pm 0.06$ & $1.11 \pm 0.07$ & $1.4 \pm 0.24$ & $2.58 \pm 0.22$ \\
\hline 24 & 7 & 24.3 & $1.49 \pm 0.08$ & $1.22 \pm 0.01$ & $1.53 \pm 0.20$ & $2.72 \pm 0.10$ \\
\hline 25 & 8 & 25.4 & $1.53 \pm 0.08$ & $1.28 \pm 0.01$ & $1.59 \pm 0.12$ & $2.80 \pm 0.14$ \\
\hline 26 & 15 & 26.3 & $1.62 \pm 0.12$ & $1.29 \pm 0.07$ & $1.85 \pm 0.21$ & $3.02 \pm 0.15$ \\
\hline 27 & 8 & 27.1 & $1.69 \pm 0.09$ & $1.33 \pm 0.07$ & $1.93 \pm 0.14$ & $3.30 \pm 0.17$ \\
\hline 28 & 7 & 28.3 & $1.74 \pm 0.12$ & $1.40 \pm 0.06$ & $1.99 \pm 0.16$ & $3.47 \pm 0.17$ \\
\hline 29 & 7 & 29.3 & $1.78 \pm 0.01$ & $1.51 \pm 0.08$ & $2.20 \pm 0.25$ & $3.48 \pm 0.18$ \\
\hline 30 & 5 & 30.4 & $2.01 \pm 0.18$ & $1.62 \pm 0.07$ & $2.76 \pm 0.36$ & $3.89 \pm 0.35$ \\
\hline
\end{tabular}

Abbreviations: AP, anteroposterior; CC, craniocaudal; SD; standard deviation; TCD, transcerebellar diameter

${ }^{\mathrm{a}}$ Values are expressed as mean $\pm \mathrm{SD}$.

Table 2. Predicted Transcerebellar, Craniocaudal and Anteroposterior Diameters by Gestational Age (5th, 50th, and 95th Centiles) and Distribution of Gestational Ages of the Study Group

\begin{tabular}{|c|c|c|c|c|c|c|c|c|c|c|c|c|c|}
\hline \multirow[t]{2}{*}{ Gestational Age, w } & \multirow[b]{2}{*}{$\mathbf{n}$} & \multicolumn{3}{|c|}{$\mathrm{TCD}, \mathbf{c m}$} & \multicolumn{3}{|c|}{ CC Diameter, cm } & \multicolumn{3}{|c|}{ AP Diameter, cm } & \multicolumn{3}{|c|}{ Surface Area, $\mathbf{c m}^{2}$} \\
\hline & & $5 \%$ & $50 \%$ & $95 \%$ & $5 \%$ & $50 \%$ & $95 \%$ & $5 \%$ & $50 \%$ & $95 \%$ & $5 \%$ & $50 \%$ & $95 \%$ \\
\hline 17 & 6 & 1.55 & 1.71 & 1.76 & 0.70 & 0.80 & 0.86 & 0.52 & 0.59 & 0.82 & 0.36 & 0.42 & 0.54 \\
\hline 18 & 9 & 1.66 & 1.80 & 1.88 & 0.75 & 0.85 & 0.98 & 0.52 & 0.69 & 0.82 & 0.33 & 0.51 & 0.59 \\
\hline 19 & 7 & 1.79 & 2.01 & 2.03 & 0.89 & 0.99 & 1.13 & 0.71 & 0.79 & 0.85 & 0.55 & 0.74 & 1.04 \\
\hline 20 & 10 & 1.93 & 2.14 & 2.22 & 0.97 & 1.03 & 1.20 & 0.78 & 0.85 & 0.91 & 0.71 & 0.84 & 1.08 \\
\hline 21 & 11 & 2.14 & 2.23 & 2.31 & 0.99 & 1.19 & 1.24 & 0.89 & 0.95 & 1.01 & 0.79 & 0.90 & 1.12 \\
\hline 22 & 9 & 2.19 & 2.37 & 2.58 & 1.10 & 1.30 & 1.48 & 0.94 & 1.04 & 1.20 & 0.91 & 1.27 & 1.52 \\
\hline 23 & 8 & 2.30 & 2.59 & 2.85 & 1.34 & 1.40 & 1.49 & 1.03 & 1.09 & 1.23 & 0.98 & 1.45 & 1.64 \\
\hline 24 & 7 & 2.62 & 2.69 & 2.93 & 1.38 & 1.51 & 1.58 & 1.07 & 1.23 & 1.32 & 1.28 & 1.43 & 1.77 \\
\hline 25 & 8 & 2.68 & 2.76 & 3.07 & 1.39 & 1.55 & 1.65 & 1.13 & 1.29 & 1.46 & 1.45 & 1.57 & 1.82 \\
\hline 26 & 15 & 2.75 & 3.04 & 3.26 & 1.42 & 1.63 & 1.75 & 1.19 & 1.28 & 1.47 & 1.56 & 1.86 & 2.17 \\
\hline 27 & 8 & 3.04 & 3.30 & 3.55 & 1.50 & 1.70 & 1.80 & 1.27 & 1.35 & 1.48 & 1.72 & 1.93 & 2.19 \\
\hline 28 & 7 & 3.21 & 3.51 & 3.67 & 1.52 & 1.77 & 1.87 & 1.34 & 1.38 & 1.5 & 1.86 & 1.92 & 2.30 \\
\hline 29 & 7 & 3.22 & 3.45 & 3.83 & 1.66 & 1.75 & 1.94 & 1.42 & 1.55 & 1.59 & 1.91 & 2.34 & 2.51 \\
\hline 30 & 5 & 3.57 & 3.89 & 4.42 & 1.79 & 2.00 & 2.29 & 1.50 & 1.62 & 1.69 & 2.46 & 2.59 & 3.36 \\
\hline
\end{tabular}

Abbreviations: AP, anteroposterior; CC, craniocaudal; TCD, transcerebellar diameter.

Bromley et al. evaluated the vermis by transabdominal ultrasound and reported that the vermis was open in 14,15 and 16 weeks in 56\%, 23\% and $13 \%$ of the fetuses, respectively implying that the postero-inferior aspect of the vermis may remain open until 17.5 weeks of gestation (13). Some researchers suggest this condition is related to the superior to inferior growth pattern of the vermis and the late fenestration of Blake's pouch (14). Therefore, the diagnosis of vermian agenesis should be avoided before 18 weeks of gestation. This was the reason why we chose to start measuring the cerebellar vermis diameter from 18 weeks of gestation in the present study (12). In agreement with the literature, we found that the cerebellum and the vermis have a linear growth from 18 weeks through 30 weeks $(3,4,14,15)$.

Evaluation of the fetal posterior fossa is performed routinely on the axial planes (5). Serial axial planes with slight angulations between them are particularly useful in 


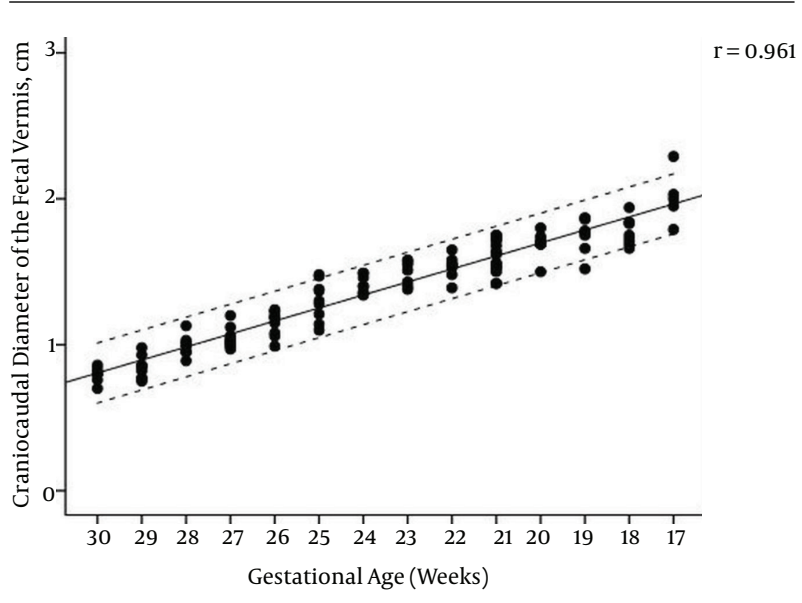

Figure 2. Scatterplot of craniocaudal diameter of the fetal vermis vs. gestational age. The lower, middle and upper lines represent the 5th, 50th, and 95th centiles.

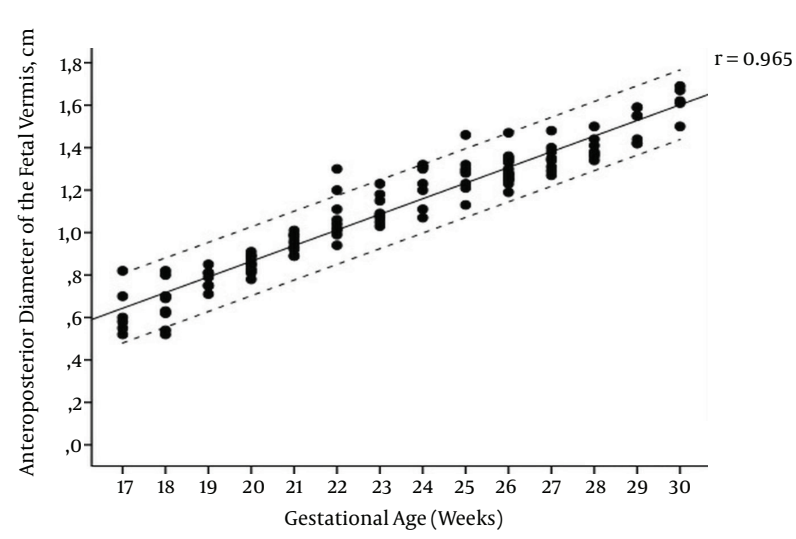

Figure 3. Scatterplot of anteroposterior diameter of the fetal vermis vs. gestational age. The lower, middle and upper lines represent the 5th, 50th and 95th centiles.

the detection of vermian anomalies such as Dandy-Walker complex (16). Additional use of the oblique coronal planes may be helpful in some cases, but this can cause the false appearance of an enlarged cisterna magna or even partial agenesis of the cerebellar vermis (17). Thus, some researchers report that it may be impossible to correctly diagnose a defect in the cerebellar vermis antenatally in all of the cases (18). Furthermore, the thin membrane separating the fourth ventricle and cisterna magna is difficult to see on ultrasound. Therefore, it seems like there is a communication between them, and the vermis may appear to be 'open' (3). This results in discrepancies between the prenatal sonographic findings and autopsies for vermian anomalies (8).

The key point of sonographic diagnosis of the defects in the cerebellar vermis is the accuracy of the scanning

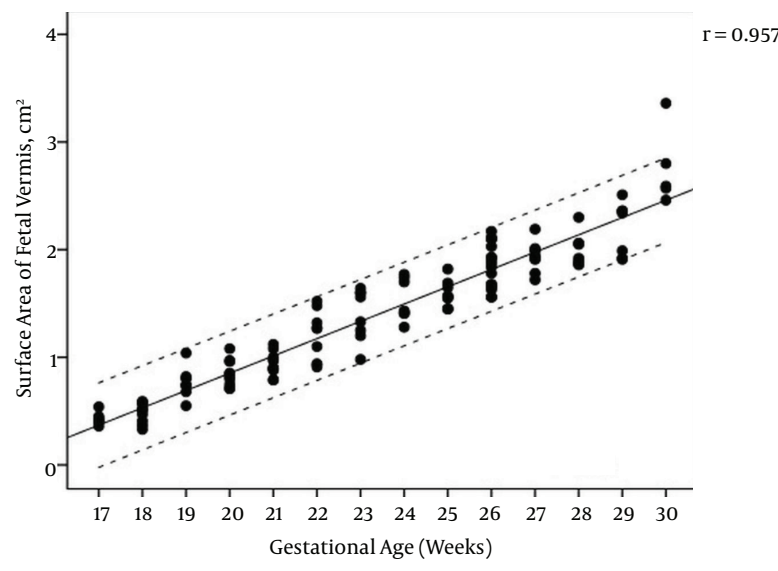

Figure 4. Scatterplot of surface area of the fetal vermis vs. gestational age. The lower middle and upper lines represent the 5th, 50th, and 95th centiles.

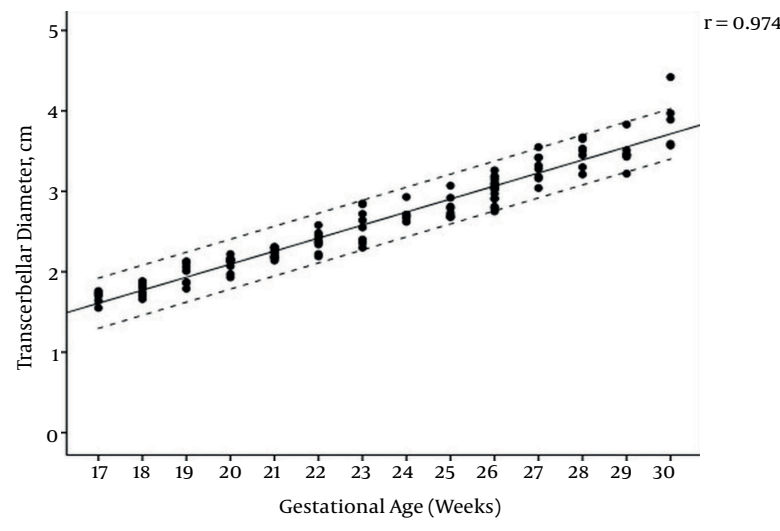

Figure 5. Scatterplot of transcerebellar diameter vs. gestational age. The lower, middle and upper lines represent the 5th, 50th, and 95th centiles.

plane. The sagittal planes must be used to evaluate the vermian integrity sufficiently. The scanning plan with corpus callosum aligned to the vermis, which manifests itself as a hyperechoic structure between the cerebellar hemispheres is used to determine the true sagittal plane with two-dimensional(2D) ultrasound. However, obtaining precise sagittal views with 2D ultrasound can sometimes be difficult according to the intrauterine fetal position. Although the transvaginal approach with 2D ultrasound in fetuses with the vertex position is a solution, magnetic resonance imaging with high resolution in sagittal planes or three-dimensional (3D) ultrasound are commonly preferred choices to overcome this problem $(15,19)$.

We agree with Pilu et al. in terms of difficulty in obtaining sagittal planes especially with 2D ultrasound (18). Even so, in this study we were not able to use either 3D ul- 
Table 3. Ratios of Transcerebellar Diameter to Vermian Diameters at Each Gestational Week ${ }^{\mathrm{a}}$

\begin{tabular}{lccc}
\hline $\begin{array}{l}\text { Gestational } \\
\text { Age, } \mathbf{w}\end{array}$ & $\begin{array}{c}\text { TCD/CC } \\
\text { Diameter }\end{array}$ & $\begin{array}{c}\text { TCD/AP } \\
\text { Diameter }\end{array}$ & $\begin{array}{c}\text { TCD/Surface } \\
\text { Area }\end{array}$ \\
\hline $\mathbf{1 7}$ & $2.1 \pm 0.2$ & $2.7 \pm 0.4$ & $4.0 \pm 0.5$ \\
$\mathbf{1 8}$ & $2.1 \pm 0.2$ & $2.7 \pm 0.4$ & $3.8 \pm 0.8$ \\
\hline $\mathbf{1 9}$ & $2.0 \pm 0.2$ & $2.5 \pm 0.2$ & $2.6 \pm 0.4$ \\
\hline $\mathbf{2 0}$ & $2.0 \pm 0.2$ & $2.5 \pm 0.2$ & $2.5 \pm 0.4$ \\
\hline $\mathbf{2 1}$ & $1.9 \pm 0.1$ & $2.4 \pm 0.1$ & $2.4 \pm 0.3$ \\
\hline $\mathbf{2 2}$ & $1.8 \pm 0.2$ & $2.2 \pm 0.2$ & $2.0 \pm 0.4$ \\
\hline $\mathbf{2 3}$ & $1.8 \pm 0.2$ & $2.3 \pm 0.2$ & $1.9 \pm 0.5$ \\
\hline $\mathbf{2 4}$ & $1.8 \pm 0.1$ & $2.2 \pm 0.2$ & $1.8 \pm 0.2$ \\
\hline $\mathbf{2 5}$ & $1.8 \pm 0.1$ & $2.2 \pm 0.1$ & $1.8 \pm 0.1$ \\
\hline $\mathbf{2 6}$ & $1.9 \pm 0.1$ & $2.3 \pm 0.1$ & $1.6 \pm 0.2$ \\
\hline $\mathbf{2 7}$ & $2.0 \pm 0.1$ & $2.4 \pm 0.2$ & $1.7 \pm 0.2$ \\
\hline $\mathbf{2 8}$ & $2.0 \pm 0.2$ & $2.5 \pm 0.2$ & $1.8 \pm 0.2$ \\
\hline $\mathbf{2 9}$ & $2.0 \pm 0.1$ & $2.3 \pm 0.1$ & $1.6 \pm 0.2$ \\
\hline $\mathbf{3 0}$ & $1.9 \pm 0.2$ & $2.4 \pm 0.2$ & $1.4 \pm 0.1$ \\
\hline & & & \\
\hline
\end{tabular}

Abbreviations: AP, anteroposterior; CC, craniocaudal; SD; standard deviation; TCD, transcerebellar diameter.

${ }^{\mathrm{a}}$ Values are expressed as mean $\pm \mathrm{SD}$.

trasound or transvaginal approach since most of the cases refused transvaginal examination because they believed it would be harmful to the fetus. Consequently, we were unable to obtain an adequate demonstration of the vermis in $26 \%$ of the cases in the vertex position and $17 \%$ in the non-vertex position, but the results were statistically significant between the two different groups of intrauterine position. Our overall measurement failure rate is concordant with the results of previous studies that reported a range of $5 \%-35.4 \%(3,4)$. However, the studies with 3D ultrasound revealed measurement success rates between $96.7 \%$ and $99.4 \%$, which is far more than our results $(4,20)$.

Since vermian abnormality is the key feature of posterior fossa defects, clinicians must evaluate the dimensions of the vermis. However, even with the ideal scanning plans and normal biometric values, subtle defects in the cerebellum and the vermis cannot be ruled out. For example, existence of the primary fissure is one of the most important features of a morphologically normal vermis, but it can be detected sonographically from 22 to 23 weeks and afterwards (21). These limitations should be shared with patients undergoing fetal anomaly screening.

The present study had some limitations. First, we did not use 3D ultrasound to compare with 2D ultrasound results. Second, our sample size was relatively small. More study participants would lend our evaluations greater power. The failure rate might also affect the power of our study by decreasing the number of sample size. This limitation could be avoided by allowing extra time for the fetus to change to a more favorable position. Third, high accuracy measurements are required because inaccurate measurements may lead to the false diagnosis of vermian agenesis. Nevertheless, the prospective nature of the study design was the strength of our study. Also, it was an advantage to be able to perform measurement of every ultrasound image by the same expert in a single institution. In addition, this is the first investigation in Turkey in which nomograms for fetal cerebellar dimensions have been constructed using $2 \mathrm{D}$ ultrasound in the midsagittal cranial plane. Therefore, these data can provide useful reference values for characterization of fetal cerebellar abnormalities in our population.

In conclusion, we created a nomogram for cerebellar vermis considering circumstances such as the absence of a 3D ultrasound and unwilling patients for transvaginal ultrasound. We hope that this study will contribute to the understanding of the developing vermis and to evaluation of the posterior fossa.

\section{Acknowledgments}

We would like to acknowledge Muzaffer Bilgin for assistance with statistical analysis.

\section{Footnotes}

Authors' Contribution: Study concept and design: Cenk Gezer and Atalay Ekin; acquisition of data: Cenk Gezer, Atalay Ekin, and Naciye Sinem Gezer; analysis and interpretation of data: Cenk Gezer and Atalay Ekin; drafting of the manuscript: Naciye Sinem Gezer; critical revision of the manuscript for important intellectual content: Cenk Gezer and Naciye Sinem Gezer; statistical analysis: Ulas Solmaz; administrative, technical, and material support: Mehmet Ozeren; and study supervision: Mehmet Ozeren.

Financial Disclosure: The authors declare no financial interests related to the material in the manuscript.

Declaration of Interests: The authors declare no conflict of interests.

\section{References}

1. Altman NR, Naidich TP, Braffman BH. Posterior fossa malformations. AJNR Am J Neuroradiol. 1992;13(2):691-724. [PubMed: 1566724].

2. Goldstein RB, Podrasky AE, Filly RA, Callen PW. Effacement of the fetal cisterna magna in association with myelomeningocele. Radiology. 1989;172(2):409-13. doi: 10.1148/radiology.172.2.2664865. [PubMed: 2664865]. 
3. Zalel Y, Seidman DS, Brand N, Lipitz S, Achiron R. The development of the fetal vermis: an in-utero sonographic evaluation. Ultrasound $\mathrm{Ob}$ stet Gynecol. 2002;19(2):136-9. doi: 10.1046/j.0960-7692.2001.00621.x. [PubMed: 11876804].

4. Zhao D, Liu W, Cai A, Li J, Chen L, Wang B. Quantitative evaluation of the fetal cerebellar vermis using the median view on three-dimensional ultrasound. Prenat Diagn. 2013;33(2):153-7. doi: 10.1002/pd.4027. [PubMed: 23238967].

5. Pilu G, Nicolaides KH. Diagnosis of fetal abnormalities: The 18-23-week scan. Taylor \& Francis; 1999.

6. Boddaert N, Klein O, Ferguson N, Sonigo P, Parisot D, Hertz-Pannier $\mathrm{L}$, et al. Intellectual prognosis of the Dandy-Walker malformation in children: the importance of vermian lobulation. Neuroradiology. 2003;45(5):320-4. doi: 10.1007/s00234-003-0980-6. [PubMed: 12682795].

7. Aletebi FA, Fung KF. Neurodevelopmental outcome after antenatal diagnosis of posterior fossa abnormalities. J Ultrasound Med. 1999;18(10):683-9. [PubMed:10511300].

8. Carroll SG, Porter H, Abdel-Fattah S, Kyle PM, Soothill PW. Correlation of prenatal ultrasound diagnosis and pathologic findings in fetal brain abnormalities. Ultrasound Obstet Gynecol. 2000;16(2):149-53. doi: 10.1046/j.1469-0705.2000.00199.x. [PubMed: 11117085].

9. Achiron R, Kivilevitch Z, Lipitz S, Gamzu R, Almog B, Zalel Y. Development of the human fetal pons: in utero ultrasonographic study. Ultrasound Obstet Gynecol. 2004;24(5):506-10. doi: 10.1002/uog.1731. [PubMed: 15459939].

10. Kennedy A. In: Diagnostic imaging: Obstetrics. Woodward PJ, editor. Salt Lake City: Amirsys; 2011. pp. 24-9.Approach to the Posterior Fossa.

11. Kollias SS, Ball WJ, Prenger EC. Cystic malformations of the posterior fossa: differential diagnosis clarified through embryologic analysis. Radiographics. 1993;13(6):1211-31. doi: 10.1148/radiographics.13.6.8031352. [PubMed: 8031352].

12. Bordarier C, Aicardi J. Dandy-Walker syndrome and agenesis of the cerebellar vermis: diagnostic problems and genetic counselling. Dev Med Child Neurol. 1990;32(4):285-94. [PubMed: 2185113].
13. Bromley B, Nadel AS, Pauker S, Estroff JA, Benacerraf BR. Closure of the cerebellar vermis: evaluation with second trimester US. Radiology. 1994;193(3):761-3. doi: 10.1148/radiology.193.3.7972820. [PubMed: 7972820].

14. Paladini D, Volpe P. Posterior fossa and vermian morphometry in the characterization of fetal cerebellar abnormalities: a prospective three-dimensional ultrasound study. Ultrasound Obstet Gynecol. 2006;27(5):482-9. doi: 10.1002/uog.2748. [PubMed: 16619375].

15. Vinals F, Munoz M, Naveas R, Shalper J, Giuliano A. The fetal cerebellar vermis: anatomy and biometric assessment using volume contrast imaging in the C-plane (VCI-C). Ultrasound Obstet Gynecol. 2005;26(6):622-7. doi: 10.1002/uog.2606. [PubMed:16254881].

16. Pilu G, Romero R, De Palma L, Jeanty P, Burdine C, Hobbins JC. Ultrasound investigation of the posterior fossa in the fetus. Am J Perinatol. 1987;4(2):155-9. doi: 10.1055/s-2007-999762. [PubMed: 3551976].

17. Laing FC, Frates MC, Brown DL, Benson CB, Di Salvo DN, Doubilet PM Sonography of the fetal posterior fossa: false appearance of megacisterna magna and Dandy-Walker variant. Radiology. 1994;192(1):24751. doi: 10.1148/radiology.192.1.8208946. [PubMed: 8208946].

18. Pilu G, Visentin A, Valeri B. The Dandy-Walker complex and fetal sonography. Ultrasound Obstet Gynecol. 2000;16(2):115-7. doi: 10.1046/j.1469-0705.2000.00197.x. [PubMed:11117078].

19. Barkovich AJ, Kjos BO, Norman D, Edwards MS. Revised classification of posterior fossa cysts and cystlike malformations based on the results of multiplanar MR imaging. AJR Am J Roentgenol. 1989;153(6):1289-300. doi: 10.2214/ajr.153.6.1289. [PubMed: 2816648].

20. Rizzo G, Pietrolucci ME, Mammarella S, Dijmeli E, Bosi C, Arduini D. Assessment of cerebellar vermis biometry at 18-32 weeks of gestation by three-dimensional ultrasound examination. J Matern Fetal Neonatal Med. 2012;25(5):519-22. doi: 10.3109/14767058.2011.622006. [PubMed: 21919549].

21. Zalel Y, Yagel S, Achiron R, Kivilevich Z, Gindes L. Three-dimensional ultrasonography of the fetal vermis at 18 to 26 weeks' gestation: time of appearance of the primary fissure. JUltrasound Med. 2009;28(1):1-8. [PubMed: 19106349]. 